\title{
Interplay between collective and single particle excitations around neutron-rich doubly-magic nuclei
}

\author{
S. LEONI
}

\begin{abstract}
Università degli Studi di Milano and INFN, Sezione di Milano, Milano, Italy
\end{abstract}

\begin{abstract}
The excitation spectra of nuclei with one or two particles outside a doubly-magic core are expected to be dominated, at low energy, by the couplings between phonon excitations of the core and valence particles. A survey of the experimental situation is given for some nuclei lying in close proximity of neutron-rich doubly-magic systems, such as ${ }^{47,49} \mathrm{Ca}$, ${ }^{133} \mathrm{Sb}$ and ${ }^{210} \mathrm{Bi}$. Data are obtained with various types of reactions (multinucleon transfer with heavy ions, cold neutron capture and neutron induced fission of ${ }^{235} \mathrm{U}$ and ${ }^{241} \mathrm{Pu}$ targets), with the employment of complex detection systems based on HPGe arrays. A comparison with theoretical calculations is also presented, in terms of large shell model calculations and of a phenomenological particle-phonon model. In the case of ${ }^{133} \mathrm{Sb}$, a new microscopic "hybrid" model is introduced: it is based on the coupling between core excitations (both collective and non-collective) of the doubly-magic core and the valence nucleon, using the Skyrme effective interaction in a consistent way.
\end{abstract}

\section{Introduction}

One of the greatest challenges of contemporary nuclear physics is the achievement of a unified microscopic description of all nuclei through the entire periodic table, on basis of the common symmetry principle of the nuclear 
force. A major difficulty is related to the very large number of nuclear configurations one has to deal with, when distributing nucleons in the available orbitals of the proton and neutron shells. As an example, in an $\mathrm{N}=\mathrm{Z}$ nucleus like ${ }^{44} \mathrm{Ti}$ such a number is of the order of $10^{4}$, and it increases quickly to $10^{10}$ in ${ }^{56} \mathrm{Ni}$ and $10^{28}$ in ${ }^{88} \mathrm{Ru}$ : this makes microscopic calculations computationally challenging in medium mass nuclei and simply impossible in heavy systems. As a consequence, different theoretical approaches have been developed to describe the nuclear many body quantum system [1]. In particular, large scale Shell Model calculations can be performed for nuclei up to mass 100 and around closed shells, assuming a frozen core as a truncation scheme. Following this approach, one restricts excitations to valence nucleons within one/two oscillator shells, neglecting almost completely the excitations of the core. This become a relevant limitation particularly in nuclei with one or two particles outside a doubly-magic core, since in these systems the lowest structure should be dominated by the couplings between phonon excitations of the core (with a high degree of collectivity) and valence particles, giving rise to series of multiplets [2]. The identification of these multiplets can provide precise, quantitative information on the phonon-particle couplings, a phenomen of primary importance, being at the origin of the quenching of spectroscopic factors [3] and of the damping of giant resonances $[4,5]$.

This paper will present the experimental situation in nuclei lying in close proximity of doubly-magic systems, such as ${ }^{47,49} \mathrm{Ca}[6,7],{ }^{133} \mathrm{Sb}[8]$ and ${ }^{210} \mathrm{Bi}[8]$. Additional examples around semi-magic systems can be found in Refs. $[9,10]$. Various types of reactions will be discussed: from multinucleon transfer with heavy ions, to cold neutron capture and neutron induced fission of ${ }^{235} \mathrm{U}$ and ${ }^{241} \mathrm{Pu}$ targets, using complex detection systems based on HPGe arrays coupled to magnetic spectrometers or fast $\mathrm{LaBr}_{3}$ scintillator detector arrays for lifetime measurements. The experimental data will be then compared with theoretical calculations either based on a phenomenological particle-phonon coupling approach [2] or on a shell model employing realistic effective nucleon-nucleon interactions. A newly developed microscopic model which takes into account couplings between the valence particle and core excitations (both collective and non-collective), using the Skyrme effective interaction in a consistent way, will be also presented in connection with the interpretation of ${ }^{133} \mathrm{Sb}$. 


\section{Around the stable doubly-magic ${ }^{48} \mathrm{Ca}$ : the cases of ${ }^{47,49} \mathbf{C a}$}

The nucleus ${ }^{48} \mathrm{Ca}$ is a double shell closure at the valley of stability, exhibiting only one sizable collective excitation, the $3^{-}$phonon at $4.507 \mathrm{MeV}$ $\left(\mathrm{B}(\mathrm{E} 3)=6.8 \pm 1 \mathrm{~W} . \mathrm{u}\right.$.), while the $2^{+}$state at $3.832 \mathrm{MeV}$ is little collective $\left(\mathrm{B}(\mathrm{E} 2)<2 \mathrm{~W} . \mathrm{u}\right.$.). The collective character of the $3^{-}$suggests the existence of multiplets of positive parity states arising by coupling the $3^{-}$excitation to the unpaired $\mathrm{p}_{3 / 2}$ neutron of ${ }^{49} \mathrm{Ca}$ and the $\mathrm{f}_{7 / 2}^{-1}$ neutron hole of ${ }^{47} \mathrm{Ca}$. Experimentally, candidates for this type of states were identified, in the past, both in ${ }^{49} \mathrm{Ca}$ and ${ }^{47} \mathrm{Ca}$, in low resolution particle spectroscopy studies [11] and in $\gamma$-spectroscopy analysis of heavy-ion transfer reactions with thick targets [12]. However, only recently firm spin and parity assignments and lifetimes of these states were obtained in experiments performed at Legnaro National Laboratory of INFN $[6,7]$. The nuclei of interest were populated by a ${ }^{48} \mathrm{Ca}$ beam $(\approx 6 \mathrm{MeV} / \mathrm{A})$ impinging on a thin ${ }^{64} \mathrm{Ni}$ target, employing the detection setup consisting of the large acceptance magnetic spectrometer PRISMA combined with the high efficiency Ge array CLARA [13].

Figure 1 shows, in the bottom, the $\gamma$-spectrum measured in coincidence with ${ }^{49} \mathrm{Ca}$ ions detected in PRISMA. All strong transitions correspond to decays from states identified as single particle excitations, besides the 660and $3357-\mathrm{keV}$ lines which depopulate the levels at $4017 \mathrm{keV}$ and $3357 \mathrm{keV}$, with $9 / 2^{+}$and $7 / 2^{-}$tentative spin assignment, respectively [6]. The 4017 $\mathrm{keV}$ state is expected to originate from the $\mathrm{p}_{3 / 2}$ neutron coupled to the $3^{-}$phonon of ${ }^{48} \mathrm{Ca}$. The spin assignments were proven by measuring the angular distribution and polarization of both transitions, which provided values consistent with electric stretched dipole for the $660-\mathrm{keV}$ line and electric stretched quadrupole for the $3357-\mathrm{keV} \gamma$ ray (cf. Fig. 1 a) and b)). In addition, the nature of these states was established by a lifetime analysis performed with the differential plunger technique, using the reaction ${ }^{48} \mathrm{Ca}+{ }^{208} \mathrm{~Pb}$ (see Fig. $1 \mathrm{c}$ ) and d)). In particular, the lifetime of the 4017 $\mathrm{keV}$ level (depopulated by the $660-\mathrm{keV} \gamma$-ray) was found to be $8.5 \pm 2.0 \mathrm{ps}$, which gives a reduced transition probability $\mathrm{B}(\mathrm{E} 3)=7.9 \pm 2.0 \mathrm{Wu}$, in good agreement with the measured strength of the $3^{-}$phonon of ${ }^{48} \mathrm{Ca}$. A similar analysis was performed on the ${ }^{47} \mathrm{Ca}$ reaction product and the state at 3999 $\mathrm{keV}$ was suggested to be the $11 / 2^{+}$or $13 / 2^{+}$member of the $3^{-} \otimes \mathrm{f}_{7 / 2}^{-1}$ multiplet. This identification was supported by the lifetime analysis, which provided a reduced transition probability equal to $\mathrm{B}(\mathrm{E} 3)=7.4 \pm 1.9$ W.u., again very similar to the $3^{-}$phonon strength of ${ }^{48} \mathrm{Ca}$. 


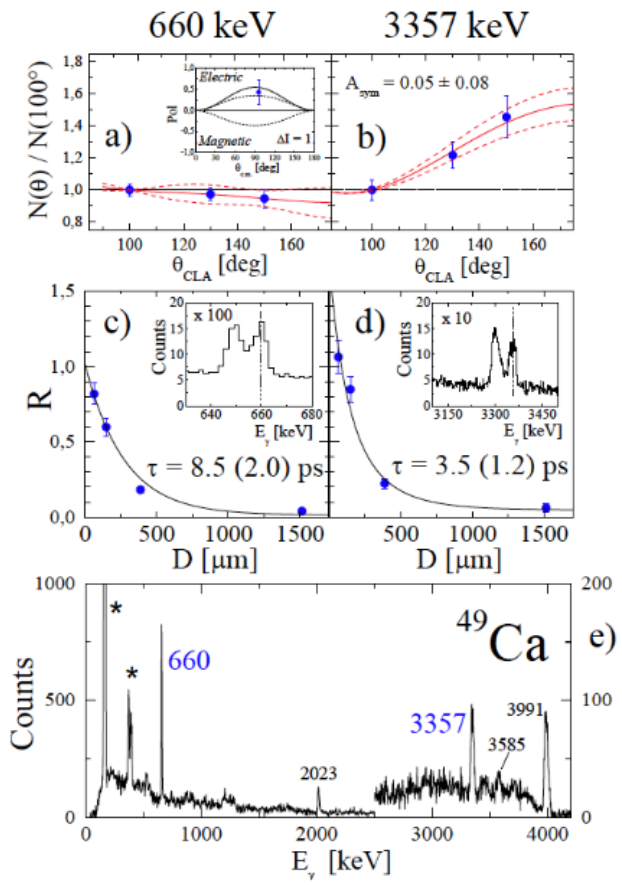

Figure 1: Panel e): $\gamma$ spectrum measured in coincidence with ${ }^{49} \mathrm{Ca}$ ions. Panels a) and b): angular distribution and polarization analysis of the 660 and $3357 \mathrm{keV}$ transitions, depopulating the $9 / 2^{+}$and $7 / 2^{-}$states. Panels c) and d): analysis of the 660 and $3357 \mathrm{keV}$ lines by the differential recoil distance Doppler shift method, providing the lifetimes of the $9 / 2^{+}$and $7 / 2^{-}$levels of ${ }^{49} \mathrm{Ca}[6]$.

A weak coupling model [2] can be applied to predict the excitation energy spectrum arising from coupling a particle/hole to a phonon. This type of calculation was performed for both ${ }^{47} \mathrm{Ca}$ and ${ }^{49} \mathrm{Ca}$, resulting in multiplets of states with energies shifted with respect to the $3^{-}$phonon, with reasonable agreement with the experimental energies, especially in the case of the $9 / 2^{+}$ state of ${ }^{49} \mathrm{Ca}$ (see Fig. 2). At the same perturbative order, the calculations predict that the reduced transition probability $\mathrm{B}(\mathrm{EL})$ to the ground state should be associated to the same multipole of the vibrational state and should have the same value of the core nucleus, as observed experimentally in both cases. This gives strong evidence that these states have a particlephonon coupled nature.

Large scale shell model calculations were also performed for ${ }^{49} \mathrm{Ca}$ using a truncated model space, since the full sd-pf (sd-pf-sdg) shell calculation is 


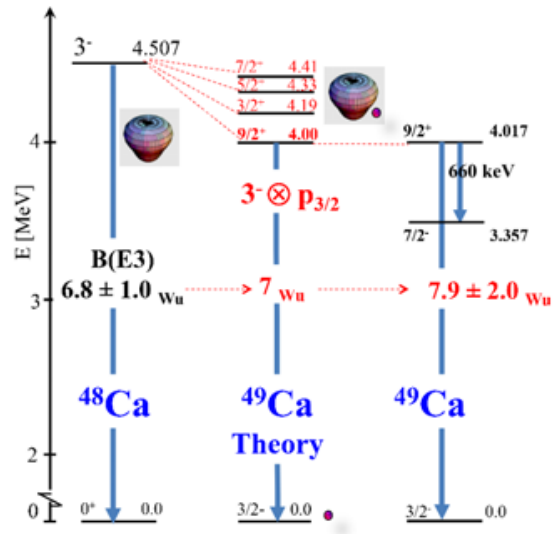

Figure 2: Right: experimental level scheme of ${ }^{49} \mathrm{Ca}$, with the $9 / 2^{+}$state as the lowest member of the $3^{-} \otimes \mathrm{p}_{3 / 2}$ multiplet, arising by coupling one neutron $\mathrm{p}_{3 / 2}$ to the collective $3^{-}$phonon of ${ }^{48} \mathrm{Ca}$ (left). The theoretical predictions (center) are obtained by a weak coupling model of Bohr and Mottelson [2] using the SkX Skyrme interaction.

still beyond the present computational capabilities [14]. By employing an effective interaction based on the monopole-based universal interaction, the energy and strength of the $3^{-}$phonon of the ${ }^{48} \mathrm{Ca}$ core are well reproduced, as well as the ones of the $9 / 2^{+}$state of ${ }^{49} \mathrm{Ca}$, while a too high spectroscopic factor $\left(0.42\right.$, with $51 \%$ contribution from the $\mathrm{g}_{9 / 2}$ orbital) was obtained in comparison with the experimental value (0.14) of Ref. [15]. All together, the shell model calculations can be considered rather successful in reproducing the ${ }^{49} \mathrm{Ca}$ data, demonstrating their capability in calculating complex core excitations up into the mass $\mathrm{A} \approx 50$ region.

\section{Around the exotic doubly-magic ${ }^{132} \mathrm{Sn}$ : the one- proton nucleus ${ }^{133} \mathrm{Sb}$}

In the case of the exotic doubly magic system ${ }^{132} \mathrm{Sn}$, three core excitations coexist, around $4-4.5 \mathrm{MeV}$, i.e. the $2^{+}, 3^{-}$and $4^{+}$phonons, with a similar strength $(\approx 7$ W.u. $)$. As a consequence, in the ${ }^{133} \mathrm{Sb}$ nucleus - with one proton outside the doubly magic ${ }^{132} \mathrm{Sn}$ core - the lowest excited states are single-proton excitations, while around $4 \mathrm{MeV}$ states arising from the coupling between core excitations and the valence proton are expected. With these characteristics, ${ }^{133} \mathrm{Sb}$ is an ideal playground for testing shell model 
predictions and/or different approaches taking into account collective excitations, in particular phonon excitations of the core. So far, the knowledge about the structure of ${ }^{133} \mathrm{Sb}$ was limited to the $\gamma$-decay of the $21 / 2^{+}$longlived isomeric state at $4545 \mathrm{keV}$ (with $\tau_{1 / 2}=16.6 \mu \mathrm{s}$ ), which prevented to easily investigate the feeding from higher-spin states $[16,17]$.

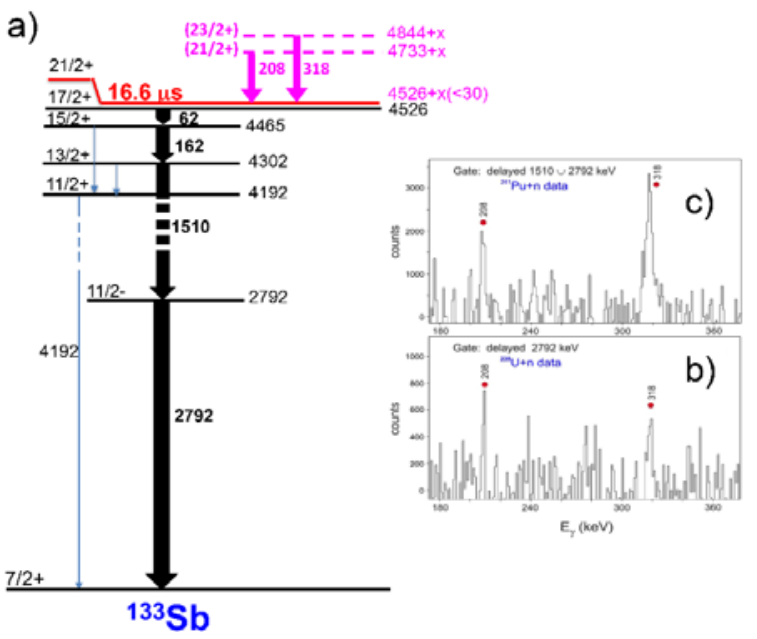

Figure 3: a) Partial level scheme of ${ }^{133} \mathrm{Sb}$, showing (in black) the known level scheme below the long lived $21 / 2^{+}$isomer $[16,17]$. New states are given in pink, with tentative spin assignments. They were observed by studying coincidences between between delayed and prompt transitions, as shown in panel b) and c) for ${ }^{235} \mathrm{U}$ and ${ }^{241} \mathrm{Pu}$ targets, respectively.

Recent $\gamma$-spectroscopy data on ${ }^{133} \mathrm{Sb}$ were obtained in the EXILL campaign at the PF1B [18] cold-neutron facility at Istitut Laue Langevine (Grenoble, France), employing an highly collimated beam with a capture flux of $10^{8}$ cold neutrons $\mathrm{s}^{-1} \mathrm{~cm}^{-2}$ on target. Two detectors setups were used, the first consisting of 8 EXOGAM clovers, 6 large coaxial detectors from GASP and 2 ILL-Clover detectors, with a total photopeak efficiency of about $6 \%$. In the second setup, the GASP and ILL detectors were replaced by $16 \mathrm{LaBr}_{3}$ crystals for lifetime measurements by fast-timing techniques. A digital data acquisition, triggerless, allowed event rates up to $0.84 \mathrm{MHz}$ to be handled and to study coincidences among $\gamma$ transitions separated in time by several tens of $\mu \mathrm{s}[19] .{ }^{133} \mathrm{Sb}$ was populated in two long runs of neutron induced fission of ${ }^{235} \mathrm{U}$ and ${ }^{241} \mathrm{Pu}$ targets. The data analysis was based on double and triple $\gamma$ coincidence histograms constructed either requiring correlations among $\gamma$-rays prompt with fission events (within $200 \mathrm{~ns}$ ) 
or requesting correlations between prompt and delayed transitions (within $20 \mu \mathrm{s}$ ). By setting gates on the strong transitions deexciting the isomer, candidates for $\gamma$-rays populating the isomer (the more intense with energies of 207 and $318 \mathrm{keV}$ ) were observed in the prompt coincidence spectrum, consistently for both fissioning targets (see Fig. $3 \mathrm{~b}$ ) and c)). These new transitions are marked in pink in the level scheme of Fig. 3 a), while in black a partial level scheme of ${ }^{133} \mathrm{Sb}$ is shown, as follows from the known $\gamma$-decay of the $16.6 \mu$ s isomer.

In the EXILL campaign, fission data from ${ }^{235} \mathrm{U}$ and ${ }^{241} \mathrm{Pu}$ targets, taken with the setup including the $\mathrm{LaBr}_{3}$ scintillators, were used to extract the lifetimes of the $13 / 2^{+}$and $15 / 2^{+}$states ${ }^{133} \mathrm{Sb}$, by fast-timing techniques [20,21]. The analysis was based on triple coincidence events, within a time window of $200 \mathrm{~ns}$, in which two $\gamma$-rays were detected in the $\mathrm{LaBr}_{3}$ scintillators and the third one in the Ge array. The half lives values $\mathrm{T}_{1 / 2}=31(8)$ ps and $<$ 20 ps were obtained for the $13 / 2^{+}$and $15 / 2^{+}$states, after averaging over the results from the ${ }^{235} \mathrm{U}$ and ${ }^{241} \mathrm{Pu}$ targets. This gives $\mathrm{B}(\mathrm{M} 1)$ values, for the $15 / 2^{+} \rightarrow 13 / 2^{+}$and $13 / 2^{+} \rightarrow 11 / 2^{+}$transitions, $>0.24 \mathrm{~W}$.u. and equal to $0.0042(15)$ W.u., respectively. Such a large difference, almost two orders of magnitude, clearly indicates that the $13 / 2^{+}$and $15 / 2^{+}$states of ${ }^{133} \mathrm{Sb}$ do not arise from similar, simple configurations. Instead, they very likely involve the coupling of the valence proton to various complex excitations of the ${ }^{132} \mathrm{Sn}$ core.

In order to interpret the experimental findings, a new microscopic model has been developed: the traditional phenomenological particle-vibration model [2] has been transformed into a "hybrid" model that includes noncollective states as well. This is very much needed, since shell model calculations in the configuration space including shells below and above the ${ }^{132} \mathrm{Sn}$ core, which must be considered to describe one-valence particle nuclei around ${ }^{132} \mathrm{Sn}$, are computationally not possible. The hybrid model calculations are self-consistent in the sense that both single-particle states and phonons come out of Hartree-Fock (HF) and Random Phase Approximation (RPA) calculations performed with the Skyrme SkX interaction introduced in Ref. [22]. In each of these subspaces one has both one-particle states and so-called one particle-one phonon states, and some of the so-called phonons turn out to be pure $1 \mathrm{p}-1 \mathrm{~h}$ states, as it can be expected. In the calculations for ${ }^{133} \mathrm{Sb}$, excitations of ${ }^{132} \mathrm{Sn}$ are considered up to $5.5 \mathrm{MeV}$ together with the proton states of the 50-82 shell. In addition, the correction for the nonorthonormality of the basis is taken into account [23]. Without any free parameter, the model does reproduce the energy sequence of the high-spin states observed experimentally, within $200 \mathrm{keV}$. Also, a gradual transition 
from more complex towards non-collective excitations is seen, with increasing spin: the importance of the proton states admixed with the low-lying $2^{+}$ decreases, while the relevance of the proton coupled with the single neutron $\mathrm{h}_{11 / 2}^{-1} \mathrm{f}_{7 / 2}$ increases. In particular, the states located, experimentally, above the $21 / 2^{+}$isomer are found to arise from almost pure (>95\%) configuration of $\pi \mathrm{g}_{7 / 2} \nu \mathrm{f}_{7 / 2} \mathrm{~h}_{11 / 2}^{-1}$ character, while a much stronger admixture is found for the $13 / 2^{+}$and $15 / 2^{+}$states, below the isomer, in agreement with the experimental results of the lifetime analysis.

At present, this hybrid model is the only tool which describes, in medium-heavy mass systems, the low-lying spectra of odd nuclei made of a magic core and an unpaired nucleon, where states having real particlevibration character co-exist with states that have less collective or noncollective character.

\section{Around the stable doubly-magic ${ }^{208} \mathrm{~Pb}$ : the one- proton, one-neutron nucleus ${ }^{210} \mathrm{Bi}$}

The ${ }^{208} \mathrm{~Pb}$ nucleus is considered one of the best known doubly-magic nuclei, therefore nuclei in its proximity are ideal candidates for testing interactions among valence particles. This is the case of ${ }^{210} \mathrm{Bi}$, a one-valence-proton, one-valence-neutron nucleus, which is expected to provide information on proton-neutron excitations and couplings of particles with excitations of the ${ }^{208} \mathrm{~Pb}$ core. The low-energy structure of ${ }^{210} \mathrm{Bi}$, up to approx. $\sim 2 \mathrm{MeV}$, should arise exclusively from couplings of a proton on the $1 h_{9 / 2}, 2 f_{7 / 2}, 1 i_{13 / 2}$, $2 f_{5 / 2}, 3 p_{3 / 2}, 3 p_{1 / 2}$ and a neutron on the $2 g_{9 / 2}, 1 i_{11 / 2}, 1 j_{15 / 2}, 3 d_{5 / 2}, 4 s_{1 / 2}$, $2 g_{7 / 2}, 3 d_{3 / 2}$ orbitals, while at higher energies states that originate from the couplings between valence particles and the very strong $3^{-}$octupole vibration of ${ }^{208} \mathrm{~Pb}$ (at $2615 \mathrm{keV}$ with $\mathrm{B}(\mathrm{E} 3) \approx 34 \mathrm{~W}$.u.) should appear.

High resolution, low spin data on ${ }^{210} \mathrm{Bi}$ were obtained during the recent EXILL campaign (discussed in Sec. 3), with the cold neutron capture reaction ${ }^{209} \mathrm{Bi}(\mathrm{n}, \gamma){ }^{210} \mathrm{Bi}[8]$. From the capture state, at $4605 \mathrm{keV}$, the $\gamma$ decay of ${ }^{210} \mathrm{Bi}$ was found to proceeds through many paths, feeding a large number of levels. This decay was investigated in great details: 64 primary $\gamma$ rays were observed (40 new) and a total number of 70 discrete states (33 new) were located below the neutron binding energy. The analysis of the angular correlations of $\gamma$ rays provided information about transitions multipolarities, which allowed to confirm most of the previously known spin-parity assignments as well as helped establishing new ones. Such a detailed experimental investigation, together with previous works [11], allows to approach a com- 
plete spectroscopy of ${ }^{210} \mathrm{Bi}$ at low spins $(\mathrm{J}<8)$. This provides a unique opportunity for a one-to-one comparison with theoretical calculations over the entire energy range, from the ground state to the neutron binding energy.

In a heavy system such as ${ }^{210} \mathrm{Bi}$, a detailed comparison with shell model calculations can only be made assuming a frozen ${ }^{208} \mathrm{~Pb}$ core. The calculations were performed by the computer code OXBASH [24], using the residual interaction of Kuo and Herling [25] with modifications introduced by Warburton and Brown (KHPE [26]). It is found that the experimental states, in large majority, correspond to excitations of one proton and one neutron valence particles. Below $2 \mathrm{MeV}$ of excitation energy the agreement between experiment and theory is very good and almost all states are reproduced by the calculations within a few $\mathrm{keV}$. This is also true above $2 \mathrm{MeV}$, where the states observed experimentally have configurations which arise from neutron and proton promotions to almost all orbitals available above the ${ }^{208} \mathrm{~Pb}$ core, allowed by the Kuo-Herling model space. Only 12 levels, above $2 \mathrm{MeV}$, do not have their theoretical counterparts, and they are most naturally considered as arising from the couplings of one-proton one-neutron excitations to the octupole vibration of the ${ }^{208} \mathrm{~Pb}$ core at $2615 \mathrm{keV}$, which is known to largely contribute to the structure of the states in neighbouring nuclei.

\section{Conclusions}

Experimental results around the doubly-magic neutron-rich cores of ${ }^{48} \mathrm{Ca}$, ${ }^{132} \mathrm{Sn}$ and ${ }^{208} \mathrm{~Pb}$ were discussed. In particular, data on ${ }^{47,49} \mathrm{Ca},{ }^{133} \mathrm{Sb}$ and ${ }^{210} \mathrm{Bi}$ were presented, focusing on the investigation of excited states arising from the coupling of valence particles/holes to core excitations: they are key ingredients for the understanding of basic phenomena, such as the quenching of spectroscopic factors and the damping mechanism in giant resonances. The present data provide an excellent testing ground for state of the art theoretical approaches, such as shell model calculations or newly developed extended microscopic particle-phonon coupling models.

\section{References}

[1] D. Lacroix, 2009 International Joliot-Curie School (EJC2009).

[2] A. Bohr, B.R. Mottelson, Nuclear Structure, I and II, W.A. Benjamin, 1975.

[3] M. B. Tsang et al., Phys. Rev. Lett. 102, 062501 (2009). 
[4] P.F. Bortignon, A. Bracco, and R. A. Broglia, Giant Resonances: Nuclear Structure at Finite Temperature (Harwood Academic, New York, 1998).

[5] Y.F. Niu et al., Phys. Rev. Lett. 114, 142501 (2015).

[6] D. Montanari et al., Phys. Lett. B 697, 288 (2011).

[7] D. Montanari et al., Phys. Rev. C 85, 044301 (2012).

[8] S. Leoni et al., Acta Phys. Pol. B 46, 637 (2015) and to be published.

[9] G. Bocchi et al., Phys. Rev. C 89, 054302 (2014).

[10] C.R. Niţă et al., Phys. Rev. C 89, 064314 (2014).

[11] Nuclear Data Base, http://www.nndc.bnl.gov/nudat2/

[12] R. Broda, J. Phys. G: Nucl. Part. Phys. 32 (2006) R151.

[13] D. Montanari, et al., Eur. Phys. J. A 47 (2011) 4 and references therein.

[14] Y. Utsuno et al., Prog. Theor. Phys. Supp. 196 (2012) 304.

[15] Y. Uozumi et al., Nucl. Phys. A 576, 123 (1994)

[16] W. Urban et al., Phys. Rev. C 62, 027301 (2000).

[17] W. Urban et al., Phys. Rev. C 79, 037304 (2009).

[18] H. Abele et al., Nucl. Instr. Meth. Phys. Res. A 562, 407 (2006).

[19] G. De France et al., Eur. Ph. J. Web of Conferences 66, 02010 (2014).

[20] J.-M. Regis et al., Nucl. Instr. Meth. Phys. Res. A 726, 191 (2013).

[21] J.-M. Regis et al., Nucl. Instr. Meth. Phys. Res. A 763, 210 (2014).

[22] B. A. Brown, Phys. Rev. C 58, 220 (1998).

[23] J. Rowe, J. Math. Phys. 10, 1774 (1969).

[24] B. A. Brown et al., MSU-NSCL Report No. 1289, 2004 (unpublished).

[25] G. Herling and T. T. S. Kuo, Nucl. Phys. A 181, 113 (1972).

[26] E. K. Warburton, B. A. Brown, Phys. Rev. C 43, 602 (1991). 